\title{
The Weierstrass elliptic function expansion method and its applications in nonlinear wave equations
}

\author{
Zhenya Yan \\ Key Laboratory of Mathematics Mechanization, Institute of systems Science, \\ AMSS, Chinese Academy of Sciences, Beijing 100080, P.R. China \\ zyyan@mmrc.iss.ac.cn
}

\begin{abstract}
In this paper, based on the close relationship between the Weierstrass elliptic function $\wp\left(\xi ; g_{2}, g_{3}\right)\left(g_{2}, g_{3}\right.$, invariants) and nonlinear ordinary differential equation, a Weierstrass elliptic function expansion method is developed in terms of the Weierstrass elliptic function instead of many Jacobi elliptic functions. The mechanism is constructive and can be carried out in computer with the aid of computer algebra (for example, Maple). Many important nonlinear wave equations arising from nonlinear science are chosen to illustrate this technique such as the new integrable Davey-Stewartsontype equation, the $(2+1)$-dimensional modified $\mathrm{KdV}$ equation, the the generalized Hirota equation in $2+1$ dimensions, the Generalized KdV equation, the (2+1)-dimensional modified Novikov-Veselov equations, $(2+1)$-dimensional generalized system of modified $\mathrm{KdV}$ equation, the coupled Klein-Gordon equation, and the (2+1)-dimensional generalization of coupled nonlinear Schrodinger equation. As a consequence, some new doubly periodic solutions are obtained in terms of the Weierstrass elliptic function. Moreover solitary wave solutions and singular solitary wave solutions are also given as simple limits of doubly periodic solutions. These solutions may be useful to explain some physical phenomena. The algorithm is also applied to other many nonlinear wave equations. Moreover we also present the general form of the technique.
\end{abstract}

PACS: 02.30.Jr; 03.65.Ge; 05.45.Yv

\section{Introduction}

It is of prime significance to investigate exact doubly periodic solutions in terms of elliptic functions, which may lead to solitary wave solutions and trigonometric function solutions in limiting cases, of nonlinear wave equations in mathematical physics[1,2]. Recently, some transformation mechanisms were developed to seek doubly periodic solutions of nonlinear wave equations in terms of the Weierstrass elliptic function[1-3], four Theta functions[1,2,4] and Jacobian elliptic functions[5-11]. But these methods are only used to seek some special types of doubly periodic solutions. There does not exist an uniform method to find all solutions of nonlinear wave equations. A natural problem is whether there exist other powerful methods to construct other types of doubly periodic solutions of nonlinear wave equations in nonlinear sciences.

In this paper, motivated by the idea in [3], we will attempt to develop an algorithm in terms of Weierstrass elliptic function $\wp\left(\xi ; g_{2}, g_{3}\right)[1,2]$ to seek new types of doubly periodic solutions of nonlinear wave equations in mathematical physics. The method is simply called the Weierstrass elliptic function expansion method and may be performed in computer with 
the aid of symbolic computation. We will choose eight nonlinear wave equations to illustrate the algorithm. These obtained results show that the algorithm is powerful to seek doubly periodic solutions of a wide class of nonlinear wave equations.

The rest of this letter is organized as follows: In section 2, we simply introduce the Weierstrass elliptic function expansion method and its algorithm. In sections 3-10, we choose ten nonlinear evolution equations to illustrate the method. As a consequence, with the aid of Maple, some new doubly periodic solutions are obtained in terms of the Weierstrass elliptic function. In addition, some special solitary wave solutions and singular solitary wave solutions are also given as simple limits of doubly periodic solutions. Finally, we also give the general form of the technique, which may be used to seek more types of doubly periodic solutions of more general nonlinear wave equations. For example (1) we may obtain non-travelling wave types of doubly periodic solutions; (2) For given variable coefficients nonlinear partial differential equation, firstly we do not need to try to reduce it to ODE.

\section{The Weierstrass elliptic function expansion method and its algorithm}

In the following we will simply introduce the method and its algorithm. Consider a given nonlinear wave equation with a physical field $u$ and two independent variables $x, t$ as

$$
F\left(u, u_{t}, u_{x}, u_{x x}, u_{x t}, u_{t t}, \ldots\right)=0,
$$

we seek its travelling wave solution $u(x, t)=u(\xi), \xi=k(x-\lambda t)$, which leads to (1) to be an nonlinear ordinary differential equation

$$
G\left(u, u^{\prime}, u^{\prime \prime}, u^{\prime \prime \prime}, \ldots\right)=0 .
$$

where the prime denotes $d / d \xi$.

Remark 1: We require that the function $G$ is a polynomial of indicated variable. If the function $G$ is not a polynomial of indicated variables, then we may use some transformations to change it into a polynomial. Otherwise the method will not work.

A crucial step of the method is to seek the power series solution of (2) in terms of the Weierstrass elliptic function

$$
\begin{gathered}
u(\xi)=u\left(\wp\left(\xi ; g_{2}, g_{3}\right)\right)=a_{0}+\sum_{i=1}^{n}\left(a_{i}\left[A \wp\left(\xi ; g_{2}, g_{3}\right)+B\right]^{\frac{i}{2}}+b_{i}\left[A \wp\left(\xi ; g_{2}, g_{3}\right)+B\right]^{-\frac{i}{2}}\right) \\
=a_{0}+\sum_{i=1}^{n}\left[a_{i}\left(\sqrt{A \wp\left(\xi ; g_{2}, g_{3}\right)+B}\right)^{i}+\frac{b_{i}}{\left(\sqrt{A \wp\left(\xi ; g_{2}, g_{3}\right)+B}\right)^{i}}\right]
\end{gathered}
$$

where $n, A \neq 0, B, a_{0}, a_{i}, b_{i}$ are parameters to be determined later, and $\wp\left(\xi ; g_{2}, g_{3}\right)$ is the Weierstrass elliptic function satisfying the nonlinear ordinary differential equation

$$
[\wp(\xi)]^{2}=4 \wp^{3}(\xi)-g_{2} \wp(\xi)-g_{3},
$$

where $g_{2}, g_{3}$ are real parameters and called invariants [1,2]. According to (4), we can derive the second order derivative of $\wp$ in the form

$$
[\wp(\xi)]^{\prime \prime}=6 \wp^{2}(\xi)-\frac{1}{2} g_{2},
$$


which is useful to solve the following nonlinear wave equations.

To determined the parameter $n$, we define a polynomial degree function as $D(u(\wp))=n$, thus we have

$$
D\left(u^{p}(\wp)\left(\frac{d^{s} u(\wp)}{d \xi^{s}}\right)^{q}\right)=n p+q(n+s) .
$$

Therefore we can determine $n$ in (3) by the leading order analysis (or balancing the highest order linear term and nonlinear terms).

Moreover, we know from (4) and (4') that

$$
\begin{gathered}
\frac{d}{d \xi} \sqrt{A \wp\left(\xi ; g_{2}, g_{3}\right)+B}=\frac{A \wp^{\prime}}{2 \sqrt{A \wp+B}}, \\
\frac{d^{2}}{d \xi^{2}} \sqrt{A \wp\left(\xi ; g_{2}, g_{3}\right)+B}=\frac{8 A^{2} \wp^{3}+12 A B \wp^{2}+A^{2} g_{3}-A B g_{2}}{4(A \wp+B) \sqrt{A \wp+B}}, \ldots \ldots \\
\frac{d}{d \xi}\left(\frac{1}{\sqrt{A \wp\left(\xi ; g_{2}, g_{3}\right)+B}}\right)=-\frac{A \wp^{\prime}}{2(A \wp+B) \sqrt{A \wp+B}}, \\
\frac{d^{2}}{d \xi^{2}}\left(\frac{1}{\sqrt{A \wp\left(\xi ; g_{2}, g_{3}\right)+B}}\right)=\frac{-12 A B \wp^{2}-2 A^{2} g_{2} \wp-A^{2} g_{3}+A B g_{2}}{4(A \wp+B)^{2} \sqrt{A \wp+B}}, \ldots \ldots
\end{gathered}
$$

therefore by substituting (3) and (4) into (2) we know that the numerator of the expression in left side of (2) becomes a polynomial about $\wp^{i}(A \wp+B)^{j / 2} \wp^{s}(i, j=0,1 ; s=0,1,2,3 \ldots)$. The conclusion is important in the procedure of solving nonlinear wave equation by using (3) and (4) as well as (4').

The above-mentioned method is summed up as follows:

Step 1: Reduce the given nonlinear wave equation (1) of two independent variables to an ODE (2) in $\xi$ by using travelling wave transformation $u(x, t)=u(\xi), \xi=k(x-\lambda t)$.

Step 2: Determine the degree $n$ in (3) by balancing the highest degree linear term and nonlinear term, and thus give the formal solution (3) (If $n$ is not a positive integer, then we firstly make the transformation $u=v^{n}$, and then we carry out the second step again.)

Step 3: Substitute (3) with the known parameter $n$ into the left side of obtained ODE (2) along with (4) and (4'), and get an expression. And then take the numerator of the expression to get a polynomial equation for $\wp^{\prime i}(A \wp+B)^{j / 2} \wp^{s}(i, j=0,1 ; s=0,1,2,3 \ldots)$.

Step 4: Set to zero the coefficients of the polynomial obtained in Step 3 to get a set of algebraic equations with respect to the unknowns $k, \lambda, A, B, g_{2}, g_{3}, a_{0}, a_{i}, b_{i}(i=1, \ldots, n)$.

Step 5: Solve the set of algebraic equations, which may not be consistent, finally derive the doubly periodic solutions of the given nonlinear equations by using the Weierstrass elliptic function.

Remark 2: In this algorithm there is no restriction at all the numbers of physical fields and variables, so one feels free to add more field(s) and independent variables whenever needed.

In the following we will apply the algorithm to many nonlinear wave equations.

\section{The new integrable Davey-Stewartson-type equation}


Consider the new integrable Davey-Stewartson-type equation[12]

$$
\begin{aligned}
& i \Psi_{\tau}+L_{1} \Psi+\Psi \Phi+\Psi \chi=0, \\
& L_{2} \chi=L_{3}|\Psi|^{2}, \\
& \Phi_{\xi}=\chi_{\eta}+\mu\left(|\Psi|^{2}\right)_{\eta}, \quad \mu=\mp 1 .
\end{aligned}
$$

where the linear differential operators are given by

$$
\begin{gathered}
L_{1}=\frac{b^{2}-a^{2}}{4} \frac{\partial^{2}}{\partial \xi^{2}}-a \frac{\partial^{2}}{\partial \xi \partial \eta}-\frac{\partial^{2}}{\partial \eta^{2}}, \\
L_{2}=\frac{b^{2}+a^{2}}{4} \frac{\partial^{2}}{\partial \xi^{2}}+a \frac{\partial^{2}}{\partial \xi \partial \eta}+\frac{\partial^{2}}{\partial \eta^{2}}, \\
L_{3}= \pm \frac{1}{4}\left(b^{2}+a^{2}+\frac{8 b^{2}(a-1)}{(a-2)^{2}-b^{2}}\right) \frac{\partial^{2}}{\partial \xi^{2}} \pm\left(a+\frac{2 b^{2}}{(a-2)^{2}-b^{2}}\right) \frac{\partial^{2}}{\partial \xi \partial \eta} \pm \frac{\partial^{2}}{\partial \eta^{2}}
\end{gathered}
$$

$a, b$ are real parameters, and $\Psi=\Psi(\xi, \eta, \tau)$ is complex while $\Phi=\Phi(\xi, \eta, \tau), \chi=\chi(\xi, \eta, \tau)$ are real. This equation was presented firstly by Maccari[12] from the Konopelchenko-Dubrovsky (KD) equation[13] by using the reduction method. (6) had been proved to be Lax integrable. Recently we obtained some other types of doubly-periodic solution in terms of Jacobi elliptic functions $[11,16]$. In the following we will use our method to investigate its other new types of doubly periodic solutions in terms of Weierstrass elliptic function.

According to the method mentioned in Section 2, we first introduce the transformations

$$
\begin{aligned}
& \Psi(\xi, \eta, \tau)=\Psi(X) \exp (i Y), \quad \Phi(\xi, \eta, \tau)=\Phi(X), \quad \chi(\xi, \eta, \tau)=\chi(X), \\
& X=k(\xi+l \eta+\lambda \tau), \quad Y=\alpha \xi+\beta \eta+\gamma \tau .
\end{aligned}
$$

where $k, l, \lambda, \alpha, \beta, \gamma$ are parameters to be determined.

From the substitution of (7) into (6), we know that if

$$
\lambda=a(\beta+\alpha l)+2 l \beta-\frac{\alpha\left(b^{2}-a^{2}\right)}{2},
$$

then (6) reduces to

$$
\begin{gathered}
k^{2} M_{1} \frac{d^{2} \Psi(X)}{d X^{2}}+M_{0} \Psi(X)+\Psi(X) \Phi(X)+\Psi(X) \chi(X)=0, \\
k^{2} M_{2} \frac{d^{2} \chi(X)}{d X^{2}}=k^{2} M_{3} \frac{d^{2} \Psi^{2}(X)}{d X^{2}}, \\
k \frac{d \Phi(X)}{d X}=k l \frac{d \chi(X)}{d X}+\mu k l \frac{d \Psi^{2}(X)}{d X},
\end{gathered}
$$

where

$$
\begin{gathered}
M_{0}=-\gamma-\frac{1}{4} \alpha^{2}\left(b^{2}-a^{2}\right)+a \alpha \beta-\beta^{2}, \\
M_{1}=\frac{b^{2}-a^{2}}{4}-a l-l^{2}, \quad M_{2}=\frac{b^{2}+a^{2}}{4}+a l+l^{2},
\end{gathered}
$$




$$
M_{3}= \pm \frac{1}{4}\left(b^{2}+a^{2}+\frac{8 b^{2}(a-1)}{(a-2)^{2}-b^{2}}\right) \pm\left(a+\frac{2 b^{2}}{(a-2)^{2}-b^{2}}\right) l \pm l^{2} .
$$

By integrating (9a) and (9b), we arrive at

$$
\chi(X)=\frac{M_{3}}{M_{2}} \Psi^{2}(X)+C_{1}, \quad \Phi(X)=\left(\frac{l M_{3}}{M_{2}}+\mu l\right) \Psi^{2}(X)+l C_{1}+C_{2},
$$

where $C_{1}, C_{2}$ are the integration constants.

To proceed further, we eliminate $\chi$ and $\Phi$ from (11), and (9a). The straightforward calculation gives rise to the nonlinear ordinary differential equation

$$
k^{2} M_{1} \frac{d^{2} \Psi(X)}{d X^{2}}+\left(M_{0}+C_{1}+l C_{1}+C_{2}\right) \Psi(X)+\frac{1}{M_{2}}\left(M_{3}+l M_{3}+\mu l M_{2}\right) \Psi^{3}(X)=0,
$$

According to the above-mentioned method, we assume that (12) has the solution in the form

$$
\Psi(X)=\Psi\left(\wp\left(X ; g_{2}, g_{3}\right)\right)=a_{0}+\sum_{i=1}^{n}\left(a_{i}\left[A \wp\left(X ; g_{2}, g_{3}\right)+B\right]^{\frac{i}{2}}+b_{i}\left[A \wp\left(X ; g_{2}, g_{3}\right)+B\right]^{-\frac{i}{2}}\right)
$$

where $\left(\wp\left(X ; g_{2}, g_{3}\right)=\wp(X)\right.$ satisfies

$$
[\wp(X)]^{\prime 2}=4 \wp^{3}(X)-g_{2} \wp(X)-g_{3},
$$

with ${ }^{\prime}:=d / d X$.

By balancing the highest degree linear term and nonlinear term, we get $n=1$. Thus (13) reduces to

$$
\Psi(X)=\Psi\left(\wp\left(X ; g_{2}, g_{3}\right)\right)=a_{0}+a_{1} \sqrt{A \wp\left(X ; g_{2}, g_{3}\right)+B}+\frac{b_{1}}{\sqrt{A \wp\left(X ; g_{2}, g_{3}\right)+B}} .
$$

With the aid of Maple, by inserting (13') into (12) along with (14) and equating the coefficients of these terms $\wp^{j}(\sqrt{A \wp+B})^{i}(i=0,1 ; j=0,1,2,3,4,5)$, we get the system of algebraic equations

$$
\begin{gathered}
\frac{1}{M_{2}}\left(M_{3}+l M_{3}+\mu l M_{2}\right)\left(12 a_{1}^{2} a_{0} B^{4}+24 a_{1} b_{1} a_{0} B^{3}+4 a_{0}^{3} B^{3}+12 b_{1}^{2} a_{0} B^{2}\right) \\
+4 a_{0} B^{3}\left(M_{0}+C_{1}+l C_{1}+C_{2}\right)=0 \\
12 a_{1}^{2} a_{0} A^{4} \frac{1}{M_{2}}\left(M_{3}+l M_{3}+\mu l M_{2}\right)=0, \\
\frac{1}{M_{2}}\left(M_{3}+l M_{3}+\mu l M_{2}\right)\left(12 a_{0}^{3} B^{2} A+24 b_{1}^{2} a_{0} A B+72 a_{1} b_{1} a_{0} B^{2} A+48 a_{1}^{2} a_{0} B^{3} A\right) \\
+12 a_{0} A B^{2}\left(M_{0}+C_{1}+l C_{1}+C_{2}\right)=0, \\
\frac{1}{M_{2}}\left(M_{3}+l M_{3}+\mu l M_{2}\right)\left(24 a_{1} b_{1} a_{0} A^{3}+4 a_{0}^{3} A^{3}+48 a_{1}^{2} a_{0} A^{3} B\right) \\
+4 a_{0} A^{3}\left(M_{0}+C_{1}+l C_{1}+C_{2}\right)=0,
\end{gathered}
$$




$$
\begin{aligned}
& \frac{1}{M_{2}}\left(M_{3}+l M_{3}+\mu l M_{2}\right)\left(72 a_{1} b_{1} a_{0} B A^{2}+12 a_{0}^{3} B A^{2}+72 a_{1}^{2} a_{0} B^{2} A^{2}+12 b_{1}^{2} a_{0} A^{2}\right) \\
& +12 a_{0} A^{2} B\left(M_{0}+C_{1}+l C_{1}+C_{2}\right)=0, \\
& \frac{1}{M_{2}}\left(M_{3}+l M_{3}+\mu l M_{2}\right)\left(12 b_{1} a_{0}^{2} B^{3}+12 b_{1} a_{1}^{2} B^{4}+12 a_{1} a_{0}^{2} B^{4}+12 a_{1} b_{1}^{2} B^{3}\right. \\
& \left.+4 a_{1}^{3} B^{5}+4 b_{1}^{3} B^{2}\right)+M_{1} k^{2}\left(b_{1} A g_{2} B^{2}-3 b_{1} A^{2} g_{3} B+a_{1} A^{2} g_{3} B^{2}-a_{1} A g_{2} B^{3}\right) \\
& +\left(M_{0}+C_{1}+l C_{1}+C_{2}\right)\left(4 b_{1} B^{3}+4 a_{1} B^{4}\right)=0, \\
& \frac{1}{M_{2}}\left(M_{3}+l M_{3}+\mu l M_{2}\right)\left(20 a_{1}^{3} A^{4} B+12 a_{1} a_{0}^{2} A^{4}+12 b_{1} a_{1}^{2} A^{4}\right) \\
& +4 a_{1} A^{4}\left(M_{0}+C_{1}+l C_{1}+C_{2}\right)+28 k^{2} M_{1} a_{1} A^{3} B=0, \\
& 4 a_{1}^{3} A^{5} \frac{1}{M_{2}}\left(M_{3}+l M_{3}+\mu l M_{2}\right)+8 k^{2} M_{1} a_{1} A^{4}=0, \\
& \frac{1}{M_{2}}\left(M_{3}+l M_{3}+\mu l M_{2}\right)\left(8 b_{1}^{3} B A+20 a_{1}^{3} A B^{4}+48 b_{1} a_{1}^{2} A B^{3}+36 b_{1} a_{0}^{2} B^{2} A\right. \\
& +48 a_{1} a_{0}^{2} A B^{3}+36 a_{1} b_{1}^{2} A B^{2}+\left(M_{0}+C_{1}+l C_{1}+C_{2}\right)\left(12 b_{1} A B^{2}+16 a_{1} A B^{3}\right) \\
& -k^{2} M_{1}\left(b_{1} A^{2} g_{2} B+2 a_{1} A^{2} g_{2} B^{2}+3 b_{1} A^{3} g_{3}-2 a_{1} A^{3} g_{3} B\right)=0, \\
& \frac{1}{M_{2}}\left(M_{3}+l M_{3}+\mu l M_{2}\right)\left(12 a_{1} b_{1}^{2} A^{3}+48 a_{1} a_{0}^{2} A^{3} B+40 a_{1}^{3} A^{3} B^{2}\right. \\
& \left.+48 b_{1} a_{1}^{2} A^{3} B+12 b_{1} a_{0}^{2} A^{3}\right)+\left(M_{0}+C_{1}+l C_{1}+C_{2}\right)\left(4 b_{1} A^{3}+16 a_{1} A^{3} B\right) \\
& -k^{2} M_{1}\left(12 b_{1} A^{2} B-32 a_{1} A^{2} B^{2}\right)=0, \\
& \frac{1}{M_{2}}\left(M_{3}+l M_{3}+\mu l M_{2}\right)\left(72 a_{1} a_{0}^{2} A^{2} B^{2}+72 b_{1} a_{1}^{2} A^{2} B^{2}+36 a_{1} b_{1}^{2} A^{2} B+40 a_{1}^{3} A^{2} B^{3}\right. \\
& \left.+4 b_{1}^{3} A^{2}+36 b_{1} a_{0}^{2} B A^{2}\right)+\left(M_{0}+C_{1}+l C_{1}+C_{2}\right)\left(24 a_{1} A^{2} B^{2}+12 b_{1} A^{2} B\right) \\
& +k^{2} M_{1}\left(a_{1} A^{4} g_{3}+12 a_{1} A B^{3}-a_{1} A^{3} g_{2} B-2 b_{1} A^{3} g_{2}-12 b_{1} A B^{2}\right)=0 \text {, }
\end{aligned}
$$

It is complicated to solve the system by hand, but with the aid of Maple, we easily get three nontrivial solutions

Case 1:

$$
\begin{gathered}
a_{0}=b_{1}=0, \quad a_{1} \neq 0, \quad A=-\frac{2 k^{2} M_{1} M_{2}}{a_{1}^{2}\left(M_{3}+l M_{3}+\mu l M_{2}\right)}, \quad B=-\frac{2 M_{2}\left(M_{0}+C_{1}+l C_{1}+C_{2}\right)}{3 a_{1}^{2}\left(M_{3}+l M_{3}+\mu l M_{2}\right)}, \\
g_{3}=-\frac{1}{27 k^{6} M_{1}^{3}}\left[4\left(M_{0}+C_{1}+l C_{1}+C_{2}\right)^{3}-9 k^{2} M_{1}^{2} g_{2}\left(M_{0}+C_{1}+l C_{1}+C_{2}\right)\right],
\end{gathered}
$$

Case 2:

$$
\begin{gathered}
a_{0}=a_{1}=0, \quad b_{1} \neq 0, \quad A=\frac{3 k^{2} M_{1} B}{M_{0}+C_{1}+l C_{1}+C_{2}}, \\
g_{2}=\frac{2 b_{1}^{2}\left(M_{0}+C_{1}+l C_{1}+C_{2}\right)\left(M_{3}+l M_{3}+\mu l M_{2}\right)+4 M_{2} B\left(M_{0}+C_{1}+l C_{1}+C_{2}\right)^{2}}{3 k^{4} M_{1}^{2} M_{2} B},
\end{gathered}
$$




$$
g_{3}=\frac{6 b_{1}^{2}\left(M_{0}+C_{1}+l C_{1}+C_{2}\right)^{2}\left(M_{3}+l M_{3}+\mu l M_{2}\right)+8 M_{2} B\left(M_{0}+C_{1}+l C_{1}+C_{2}\right)^{3}}{27 k^{6} M_{1}^{3} M_{2} B},
$$

Case 3:

$$
\begin{gathered}
a_{0}=0, \quad a_{1} n p t=0, \quad b_{1} \neq 0, \quad A=-\frac{2 k^{2} M_{1} M_{2}}{a_{1}^{2}\left(M_{3}+l M_{3}+\mu l M_{2}\right)}, \\
B=-\frac{2 M_{2}\left(M_{0}+C_{1}+l C_{1}+C_{2}\right)+6 a_{a} b_{1}\left(M_{3}+l M_{3}+\mu l M_{2}\right)}{3 a_{1}^{2}\left(M_{3}+l M_{3}+\mu l M_{2}\right)}, \\
g_{2}=\frac{1}{3 k^{4} M_{1}^{2} M_{2}^{2}}\left[33 a_{1}^{2} b_{1}^{2}\left(M_{3}+l M_{3}+\mu l M_{2}\right)^{2}+4 M_{2}^{2}\left(M_{0}+C_{1}+l C_{1}+C_{2}\right)^{2}\right. \\
\left.\quad+24 a_{1} b_{1} M_{1}\left(M_{3}+l M_{3}+\mu l M_{2}\right)\left(M_{0}+C_{1}+l C_{1}+C_{2}\right)\right], \\
g_{3}=\frac{1}{27 k^{6} M_{1}^{3} M_{2}^{3}}\left[8 M_{2}^{3}\left(M_{0}+C_{1}+l C_{1}+C_{2}\right)^{3}+189 a_{1}^{3} b_{1}^{3}\left(M_{3}+l M_{3}+\mu l M_{2}\right)^{3}\right. \\
\quad+72 a_{1} b_{1} M_{2}^{2}\left(M_{3}+l M_{3}+\mu l M_{2}\right)\left(M_{0}+C_{1}+l C_{1}+C_{2}\right)^{2} \\
+207 a_{1}^{2} b_{1}^{2} M_{2}\left(M_{3}+l M_{3}+\mu l M_{2}\right)^{2}\left(M_{0}+C_{1}+l C_{1}+C_{2}\right),
\end{gathered}
$$

Therefore according to (7), (11), (13') and (16)-(18), we get three types of Weierstrass elliptic function solutions of (1):

Solution 1:

$$
\begin{gathered}
\Psi_{1}= \pm\left(-\frac{2 k^{2} M_{1} M_{2}}{M_{3}+l M_{3}+\mu l M_{2}} \wp\left(X ; g_{2}, g_{3}\right)-\frac{2 M_{2}\left(M_{0}+C_{1}+l C_{1}+C_{2}\right)}{3\left(M_{3}+l M_{3}+\mu l M_{2}\right)}\right)^{\frac{1}{2}} \exp (i Y), \\
\chi_{1}=-\frac{2 k^{2} M_{1} M_{3}}{M_{3}+l M_{3}+\mu l M_{2}} \wp\left(X ; g_{2}, g_{3}\right)-\frac{2 M_{3}\left(M_{0}+C_{1}+l C_{1}+C_{2}\right)}{3\left(M_{3}+l M_{3}+\mu l M_{2}\right)}+C_{1}, \\
\Phi_{1}=\left(\frac{l M_{3}}{M_{2}}+\mu l\right)\left[-\frac{2 k^{2} M_{1} M_{2}}{M_{3}+l M_{3}+\mu l M_{2}} \wp\left(X ; g_{2}, g_{3}\right)-\frac{2 M_{2}\left(M_{0}+C_{1}+l C_{1}+C_{2}\right)}{3\left(M_{3}+l M_{3}+\mu l M_{2}\right)}\right] \\
+l C_{1}+C_{2},
\end{gathered}
$$

where $M_{i}(i=1,2,3), g_{3}$ are determined by (10) and (16), and $X=k(\xi+l \eta+[(\beta+\alpha l)+$ $\left.\left.2 l \beta-\frac{\alpha\left(b^{2}-a^{2}\right)}{2}\right] \tau\right), \quad Y=\alpha \xi+\beta \eta+\gamma \tau$.

Solution 2:

$$
\begin{gathered}
\Psi_{2}=b_{1}\left(\frac{3 k^{2} M_{1} B}{M_{0}+C_{1}+l C_{1}+C_{2}} \wp\left(X ; g_{2}, g_{3}\right)+B\right)^{-\frac{1}{2}} \exp (i Y), \\
\chi_{2}=\frac{b_{1}^{2} M_{3}}{M_{2}}\left(\frac{3 k^{2} M_{1} B}{M_{0}+C_{1}+l C_{1}+C_{2}} \wp\left(X ; g_{2}, g_{3}\right)+B\right)^{-1}+C_{1}, \\
\Phi_{2}=\left(\left(\frac{l M_{3}}{M_{2}}+\mu l\right)\left(\frac{3 k^{2} M_{1} B}{M_{0}+C_{1}+l C_{1}+C_{2}} \wp\left(X ; g_{2}, g_{3}\right)+B\right)^{-1}+l C_{1}+C_{2},\right.
\end{gathered}
$$


where $M_{i}(i=1,2,3), g_{2}, g_{3}$ are determined by (10) and (17), and $X=k(\xi+l \eta+[(\beta+\alpha l)+$ $\left.\left.2 l \beta-\frac{\alpha\left(b^{2}-a^{2}\right)}{2}\right] \tau\right), \quad Y=\alpha \xi+\beta \eta+\gamma \tau$.

Solution 3:

$$
\begin{gathered}
\Psi_{3}=\frac{a_{1} A \wp\left(X ; g_{2}, g_{3}\right)+a_{1} B+b_{1}}{\sqrt{A \wp\left(X ; g_{2}, g_{3}\right)+B}} \exp (i Y), \\
\chi_{3}=\frac{M_{3}\left(a_{1} A \wp\left(X ; g_{2}, g_{3}\right)+a_{1} B+b_{1}\right)^{2}}{M_{2}\left(A \wp\left(X ; g_{2}, g_{3}\right)+B\right)}+C_{1}, \\
\Phi_{3}=\left(\frac{l M_{3}}{M_{2}}+\mu l\right) \frac{\left(a_{1} A \wp\left(X ; g_{2}, g_{3}\right)+a_{1} B+b_{1}\right)^{2}}{A \wp\left(X ; g_{2}, g_{3}\right)+B}+l C_{1}+C_{2},
\end{gathered}
$$

where $M_{i}(i=1,2,3), A, B, g_{2}, g_{3}$ are determined by (10) and (18), and $X=k(\xi+l \eta+[(\beta+$ $\left.\left.\alpha l)+2 l \beta-\frac{\alpha\left(b^{2}-a^{2}\right)}{2}\right] \tau\right), \quad Y=\alpha \xi+\beta \eta+\gamma \tau$.

In order to better understand the solution (19) in a more familiar manner, we rewrite (19) as the forms in terms of Jacobi elliptic function

$$
\begin{gathered}
\Psi_{11}= \pm\left(-\frac{2 k^{2} M_{1} M_{2}\left[e_{2}-\left(e_{2}-e_{3}\right) \mathrm{cn}^{2}(R X ; m)\right]}{M_{3}+l M_{3}+\mu l M_{2}}-\frac{2 M_{2}\left(M_{0}+C_{1}+l C_{1}+C_{2}\right)}{3\left(M_{3}+l M_{3}+\mu l M_{2}\right)}\right)^{\frac{1}{2}} \exp (i Y), \\
\chi_{11}=-\frac{2 k^{2} M_{1} M_{3}\left[e_{2}-\left(e_{2}-e_{3}\right) \mathrm{cn}^{2}(R X ; m)\right]}{M_{3}+l M_{3}+\mu l M_{2}}-\frac{2 M_{3}\left(M_{0}+C_{1}+l C_{1}+C_{2}\right)}{3\left(M_{3}+l M_{3}+\mu l M_{2}\right)}+C_{1}, \\
\Phi_{11}=\left(\frac{l M_{3}}{M_{2}}+\mu l\right)\left[-\frac{2 k^{2} M_{1} M_{2}\left[e_{2}-\left(e_{2}-e_{3}\right) \mathrm{cn}^{2}(R X ; m)\right]}{M_{3}+l M_{3}+\mu l M_{2}}-\frac{2 M_{2}\left(M_{0}+C_{1}+l C_{1}+C_{2}\right)}{3\left(M_{3}+l M_{3}+\mu l M_{2}\right)}\right] \\
+l C_{1}+C_{2},
\end{gathered}
$$

where $R=\sqrt{e_{1}-e_{3}}, m^{2}=\left(e_{2}-e_{3}\right) /\left(e_{1}-e_{3}\right)$ is the modulus of the Jacobi elliptic function, $e_{i}\left(i=1,2,3 ; e_{1}>e_{2}>e_{3}\right)$ are three roots of the cubic equation $4 z^{3}-g_{2} z-g_{3}=0$.

Because when $m \rightarrow 1$, i.e., $e_{2} \rightarrow e_{1}, \operatorname{cn}(R X ; m) \rightarrow \operatorname{sech}(R X)$, thus the solitary wave profile can be written as the form

$$
\begin{gathered}
\Psi_{12}= \pm\left(-\frac{2 k^{2} M_{1} M_{2}\left[e_{1}-\left(e_{1}-e_{3}\right) \operatorname{sech}^{2}(R X)\right]}{M_{3}+l M_{3}+\mu l M_{2}}-\frac{2 M_{2}\left(M_{0}+C_{1}+l C_{1}+C_{2}\right)}{3\left(M_{3}+l M_{3}+\mu l M_{2}\right)}\right)^{\frac{1}{2}} \exp (i Y), \\
\chi_{12}=-\frac{2 k^{2} M_{1} M_{3}\left[e_{1}-\left(e_{1}-e_{3}\right) \operatorname{sech}^{2}(R X)\right]}{M_{3}+l M_{3}+\mu l M_{2}}-\frac{2 M_{3}\left(M_{0}+C_{1}+l C_{1}+C_{2}\right)}{3\left(M_{3}+l M_{3}+\mu l M_{2}\right)}+C_{1}, \\
\Phi_{12}=\left(\frac{l M_{3}}{M_{2}}+\mu l\right)\left[-\frac{2 k^{2} M_{1} M_{2}\left[e_{1}-\left(e_{1}-e_{3}\right) \operatorname{sech}^{2}(R X)\right]}{M_{3}+l M_{3}+\mu l M_{2}}-\frac{2 M_{2}\left(M_{0}+C_{1}+l C_{1}+C_{2}\right)}{3\left(M_{3}+l M_{3}+\mu l M_{2}\right)}\right] \\
+l C_{1}+C_{2},
\end{gathered}
$$

When $X \rightarrow \infty$, we know that $\left|\Psi_{12}\right|$ approaches a constant, that is, $\sqrt{-\frac{2 M_{2}\left(M_{0}+C_{1}+l C_{1}+C_{2}\right)}{3\left(M_{3}+l M_{3}+\mu l M_{2}\right)}}$. Similarly, the solutions $\Psi_{12}$ and $\chi_{12}$ also have the properties.

In addition, the Weierstrass elliptic function $\wp\left(X ; g_{2}, g_{3}\right)$ has another solution

$$
\wp\left(X ; g_{2}, g_{3}\right)=e_{3}+\left(e_{1}-e_{3}\right) \mathrm{ns}^{2}(R X ; m),
$$


where $R=\sqrt{e_{1}-e_{3}}$, and $\mathrm{ns}=\frac{1}{\mathrm{sn}}$ is the Jacobi elliptic function.

Therefore from (19) we has

$$
\begin{gathered}
\Psi_{13}= \pm\left(-\frac{2 k^{2} M_{1} M_{2}\left[e_{3}+\left(e_{1}-e_{3}\right) \mathrm{ns}^{2}(R X ; m)\right]}{M_{3}+l M_{3}+\mu l M_{2}}-\frac{2 M_{2}\left(M_{0}+C_{1}+l C_{1}+C_{2}\right)}{3\left(M_{3}+l M_{3}+\mu l M_{2}\right)}\right)^{\frac{1}{2}} \exp (i Y), \\
\chi_{13}=-\frac{2 k^{2} M_{1} M_{3}\left[e_{3}+\left(e_{1}-e_{3}\right) \mathrm{ns}^{2}(R X ; m)\right]}{M_{3}+l M_{3}+\mu l M_{2}}-\frac{2 M_{3}\left(M_{0}+C_{1}+l C_{1}+C_{2}\right)}{3\left(M_{3}+l M_{3}+\mu l M_{2}\right)}+C_{1}, \\
\Phi_{13}=\left(\frac{l M_{3}}{M_{2}}+\mu l\right)\left[-\frac{2 k^{2} M_{1} M_{2}\left[e_{3}+\left(e_{1}-e_{3}\right) \mathrm{ns}^{2}(R X ; m)\right]}{M_{3}+l M_{3}+\mu l M_{2}}-\frac{2 M_{2}\left(M_{0}+C_{1}+l C_{1}+C_{2}\right)}{3\left(M_{3}+l M_{3}+\mu l M_{2}\right)}\right] \\
+l C_{1}+C_{2},
\end{gathered}
$$

Because when $m \rightarrow 1$, i.e., we know that $e_{2} \rightarrow e_{1}, \operatorname{ns}(R X ; m) \rightarrow \operatorname{coth}(R X)$, thus the singular solitary wave profile can be written as the form

$$
\begin{gathered}
\Psi_{14}= \pm\left(-\frac{2 k^{2} M_{1} M_{2}\left[e_{3}+\left(e_{1}-e_{3}\right) \operatorname{coth}^{2}(R X)\right]}{M_{3}+l M_{3}+\mu l M_{2}}-\frac{2 M_{2}\left(M_{0}+C_{1}+l C_{1}+C_{2}\right)}{3\left(M_{3}+l M_{3}+\mu l M_{2}\right)}\right)^{\frac{1}{2}} \exp (i Y), \\
\chi_{14}=-\frac{2 k^{2} M_{1} M_{3}\left[e_{3}+\left(e_{1}-e_{3}\right) \operatorname{coth}^{2}(R X)\right]}{M_{3}+l M_{3}+\mu l M_{2}}-\frac{2 M_{3}\left(M_{0}+C_{1}+l C_{1}+C_{2}\right)}{3\left(M_{3}+l M_{3}+\mu l M_{2}\right)}+C_{1}, \\
\Phi_{14}=\left(\frac{l M_{3}}{M_{2}}+\mu l\right)\left[-\frac{2 k^{2} M_{1} M_{2}\left[e_{3}+\left(e_{1}-e_{3}\right) \operatorname{coth}^{2}(R X)\right]}{M_{3}+l M_{3}+\mu l M_{2}}-\frac{2 M_{2}\left(M_{0}+C_{1}+l C_{1}+C_{2}\right)}{3\left(M_{3}+l M_{3}+\mu l M_{2}\right)}\right] \\
+l C_{1}+C_{2},
\end{gathered}
$$

When $X \rightarrow 0$, we know that $\left|\Psi_{14}\right| \rightarrow \infty$. This shows that for some time $\tau=\tau_{0}$, there exist the curve $f_{0}(\xi, \eta)=0$, on which the solution $\left|\Psi_{14}\right|$ will below up. Similarly, the solutions $\Psi_{14}$ and $\chi_{14}$ also have the properties.

Similarly, we can also rewrite the solutions (20) and (21) as another forms in terms of Jacobi elliptic function or the hyperbolic function.

\section{The (2+1)-dimensional modified $\mathrm{KdV}$ equation}

We consider the (2+1)-dimensional modified KdV equation[14]

$$
u_{t}+u_{x x z}+\alpha u^{2} u_{z}+\beta u_{x}\left(\partial_{x}^{-1}\left(u u_{z}\right)\right)+\gamma u u_{x}\left(\partial_{x}^{-1} u_{z}\right)=0
$$

where $\alpha+\frac{1}{2} \beta+\gamma \neq 0, \partial_{x}^{-1} u=\int u d x$, and $\alpha, \beta, \gamma$ are numbers parameters. Recently Toda[14] had shown that (27) passed the Painleve test only when $\alpha, \beta, \gamma$ satisfy the conditions $\alpha=\beta \neq 0$, or $\alpha=\beta+\gamma$ and $\beta+4 \gamma \neq 0$, or $\alpha=\beta+\gamma / 2$ and $\beta+\gamma \neq 0$.

According to our method, we seek the solution in the form

$$
u(\xi)=a_{0}+a_{1} \sqrt{A \wp\left(\xi ; g_{2}, g_{3}\right)+B}+\frac{b_{1}}{\sqrt{A \wp\left(\xi ; g_{2}, g_{3}\right)+B}} .
$$


where $\xi=k(x+l z+\lambda t)$, and $k, l, \lambda$ are parameters, $\wp\left(\xi ; g_{2}, g_{3}\right)=\wp(\xi)$ satisfies

$$
[\wp(\xi)]^{\prime 2}=4 \wp^{3}(\xi)-g_{2} \wp(\xi)-g_{3},
$$

Substituting (28) into (27) along with (29) and collecting the coefficients of these terms $\wp^{i}(\sqrt{A \wp+B})^{j}(j=0,1 ; i=1, \ldots, 5)$ we get a system of algebraic equations about the unknowns $a_{0}, a_{1}, b_{1}, A, B, k, l, \lambda$. By solving the system we can determine these unknowns and their relationships. Therefore we can gain the following doubly periodic solutions in terms of the Weierstrass elliptic function:

Solution 1:

$$
u_{1}(\xi)=\sqrt{-\frac{6 k^{2}}{\alpha+\frac{1}{2} \beta+\gamma} \wp\left(\xi ; g_{2}, g_{3}\right)-\frac{\lambda}{l\left(\alpha+\frac{1}{2} \beta+\gamma\right)}},
$$

where $g_{2}$ is free and

$$
g_{3}=\frac{-4 \lambda^{3}+9 k^{4} l^{2} \lambda g_{2}}{27 k^{6} l^{3}}
$$

Solution 2:

$$
u_{2}(\xi)=\frac{b_{1}}{\sqrt{\frac{3 k^{2} l B}{\lambda} \wp\left(\xi ; g_{2}, g_{3}\right)+B}},
$$

where

$$
g_{2}=\frac{2 \lambda l b_{1}\left(\alpha+\frac{1}{2} \beta+\gamma\right)+12 \lambda^{2} B}{9 k^{4} l^{2} B}, \quad g_{3}=\frac{2 \lambda^{2} l b_{1}\left(\alpha+\frac{1}{2} \beta+\gamma\right)+8 \lambda^{3} B}{27 k^{6} l^{3} B} .
$$

Solution 3:

$$
u_{3}(\xi)=\frac{-6 k^{2} l \wp\left(\xi ; g_{2}, g_{3}\right)-2 \lambda-a_{1} b_{1} l\left(\alpha+\frac{1}{2} \beta+\gamma\right)}{\sqrt{-6 k^{2} l^{2}\left(\alpha+\frac{1}{2} \beta+\gamma\right) \wp\left(\xi ; g_{2}, g_{3}\right)-2 l\left(\alpha+\frac{1}{2} \beta+\gamma\right)}} .
$$

where

$$
\begin{gathered}
g_{2}=\frac{72 a_{1} b_{1} l\left(\alpha+\frac{1}{2} \beta+\gamma\right)+36 \lambda^{2}+33 a_{1}^{2} b_{1}^{2} l^{2}\left(\alpha+\frac{1}{2} \beta+\gamma\right)^{2}}{27 k^{4} l^{2}} \\
g_{3}=\frac{24 a_{1} b_{1} l \lambda^{2}\left(\alpha+\frac{1}{2} \beta+\gamma\right)+8 \lambda^{3}+23 a_{1}^{2} b_{1}^{2} l^{2} \lambda\left(\alpha+\frac{1}{2} \beta+\gamma\right)^{2}+7 a_{1}^{3} b_{1}^{3} l^{3}\left(\alpha+\frac{1}{2} \beta+\gamma\right)^{3}}{27 k^{3} l^{3}},
\end{gathered}
$$

\section{A generalized Hirota equation in $2+1$ dimensions}

Consider the generalized Hirota equation in $2+1$ dimensions[15]

$$
\begin{aligned}
& i \Psi_{\tau}+\Psi_{\xi \eta}+i \Psi_{\xi \xi \xi}+\Psi \Phi-i|\Psi|^{2} \Psi_{\xi}=0 \\
& 3 \Phi_{\xi}+\left(|\Psi|^{2}\right)_{\eta}=0 .
\end{aligned}
$$

where $\Psi=\Psi(\xi, \eta, \tau)$ is complex while $\Phi=\Phi(\xi, \eta, \tau)$ is real. By using the above method, we can arrive at three Weierstrass elliptic function solutions:

Solution 1:

$$
\Psi_{1}=\exp [i(\alpha \xi+\beta \eta+\gamma \tau)] \sqrt{6 k^{2} \wp\left(k(\xi+l \eta+\lambda \tau) ; g_{2}, g_{3}\right)+2\left(\lambda+\beta+\alpha l-3 \alpha^{2}\right)},
$$




$$
\begin{aligned}
\Phi_{1}=- & \frac{l}{3}\left[6 k^{2} \wp\left(k(\xi+l \eta+\lambda \tau) ; g_{2}, g_{3}\right)+2\left(\lambda+\beta+\alpha l-3 \alpha^{2}\right)\right] \\
& +\left(\gamma+\alpha \beta-\alpha^{3}\right)+(l-3 \alpha)\left(\alpha+\beta+\alpha l-3 \alpha^{2}\right),
\end{aligned}
$$

where $k, l, \lambda, \alpha, \beta, \gamma$ are constants, and $g_{3}$ satisfies

$$
g_{3}=\frac{-4\left(\lambda+\beta+\alpha l-3 \alpha^{2}\right)^{3}+9 k^{4} g_{2}\left(\lambda+\beta+\alpha l-3 \alpha^{2}\right)}{27 k^{6}} .
$$

Solution 2:

$$
\begin{aligned}
\Psi_{2}= & \frac{b_{1} \exp [i(\alpha \xi+\beta \eta+\gamma \tau)]}{\sqrt{\frac{3 k^{2} B}{\lambda+\beta+\alpha l-3 \alpha^{2}} \wp\left(k(\xi+l \eta+\lambda \tau) ; g_{2}, g_{3}\right)+B}}, \\
\Phi_{2}= & \frac{b_{1} l\left(\lambda+\beta+\alpha l-3 \alpha^{2}\right)}{3 k^{2} B \wp\left(k(\xi+l \eta+\lambda \tau) ; g_{2}, g_{3}\right)+3 B\left(\lambda+\beta+\alpha l-3 \alpha^{2}\right)} \\
& +\left(\gamma+\alpha \beta-\alpha^{3}\right)+(l-3 \alpha)\left(\alpha+\beta+\alpha l-3 \alpha^{2}\right) .
\end{aligned}
$$

where $b_{1}, k, l, \lambda, \alpha, \beta, \gamma$ are constants, and $g_{2}, g_{3}$ satisfy

$$
\begin{aligned}
& g_{2}=\frac{-2 b_{1}^{2}\left(\lambda+\beta+\alpha l-3 \alpha^{2}\right)+12 B\left(\lambda+\beta+\alpha l-3 \alpha^{2}\right)^{2}}{9 k^{4} B}, \\
& g_{3}=\frac{-6 b_{1}^{2}\left(\lambda+\beta+\alpha l-3 \alpha^{2}\right)+24 B\left(\lambda+\beta+\alpha l-3 \alpha^{2}\right)^{3}}{81 B k^{6}} .
\end{aligned}
$$

Solution 3:

$$
\begin{gathered}
\Psi_{3}=\frac{\left[6 k^{2} \wp\left(k(\xi+l \eta+\lambda \tau) ; g_{2}, g_{3}\right)+2\left(\lambda+\beta+\alpha l-3 \alpha^{2}\right)-a_{1} b_{1}\right] \exp [i(\alpha \xi+\beta \eta+\gamma \tau)]}{\sqrt{6 k^{2} \wp\left(k(\xi+l \eta+\lambda \tau) ; g_{2}, g_{3}\right)+2\left(\lambda+\beta+\alpha l-3 \alpha^{2}\right)-2 a_{1} b_{1}}}, \\
\Phi_{3}=\frac{-l\left[6 k^{2} \wp\left(k(\xi+l \eta+\lambda \tau) ; g_{2}, g_{3}\right)+2\left(\lambda+\beta+\alpha l-3 \alpha^{2}\right)-a_{1} b_{1}\right]^{2}}{3\left[6 k^{2} \wp\left(k(\xi+l \eta+\lambda \tau) ; g_{2}, g_{3}\right)+2\left(\lambda+\beta+\alpha l-3 \alpha^{2}\right)-2 a_{1} b_{1}\right]} \\
+\left(\gamma+\alpha \beta-\alpha^{3}\right)+(l-3 \alpha)\left(\alpha+\beta+\alpha l-3 \alpha^{2}\right),
\end{gathered}
$$

where $a_{1}, b_{1}, k, l, \lambda, \alpha, \beta, \gamma$ are constants, and $g_{2}, g_{3}$ satisfy

$$
\begin{gathered}
g_{2}=\frac{33 a_{1}^{2} b_{1}^{2}-72 a_{1} b_{1}\left(\lambda+\beta+\alpha l-3 \alpha^{2}\right)+36\left(\lambda+\beta+\alpha l-3 \alpha^{2}\right)^{2}}{27 k^{4}} \\
g_{3}=\frac{1}{729 k^{6}}\left[-189 a_{1}^{3} b_{1}^{3}+621 a_{1}^{2} b_{1}^{2}\left(\lambda+\beta+\alpha l-3 \alpha^{2}\right)-648 a_{1} b_{1}\left(\lambda+\beta+\alpha l-3 \alpha^{2}\right)^{2}\right. \\
\left.+216\left(\lambda+\beta+\alpha l-3 \alpha^{2}\right)^{3}\right] .
\end{gathered}
$$

\section{The Generalized KdV equation}


Consider the generalized KdV equation[18]

$$
u_{t}+\alpha u^{p} u_{x}+\beta u_{x x x}=0, \quad p>2 .
$$

We make the transform $u(x, t)=w^{2 / p}(\xi), \quad \xi=k(x+\lambda t)$. Thus (36) reduces to

$$
\lambda w^{2}+\frac{\alpha}{p+1} w^{4}+\beta k^{2} \frac{2}{p}\left(\frac{2}{p}-1\right) w^{2}+\beta k^{2} \frac{2}{p} w w^{\prime \prime}=0 .
$$

By using the above method we can get the solutions of (37). Therefore we gain two types of Weierstrass elliptic function solutions of (36):

Solution 1:

$$
u_{1}=\left(\frac{2 \beta k^{2}(p+2)}{\alpha p^{2}} \wp\left(\xi ; g_{2}, g_{3}\right)-\frac{\lambda(p+1)(p+2)}{6 \alpha}\right)^{\frac{1}{p}} \text {. }
$$

where $g_{2}, g_{3}$ satisfy

$$
g_{2}=\frac{\lambda^{2} p^{4}}{12 \beta^{2} k^{4}}, \quad g_{3}=\frac{\lambda^{3} p^{6}}{216 \beta^{3} k^{6}}
$$

Solution 2:

$$
u_{2}=\left(\frac{\left(16 \beta k^{2} \wp\left(\xi ; g_{2}, g_{3}\right)+\frac{1}{3} \lambda p^{2}\right)^{2}}{-\frac{128 \alpha \beta k^{2} p^{2}}{(2+p)(p+1)} \wp\left(\xi ; g_{2}, g_{3}\right)+\frac{16 \alpha \lambda p^{4}}{3(2+p)(p+1)}}\right)^{\frac{1}{p}}
$$

where $g_{2}, g_{3}$ satisfy

$$
g_{2}=\frac{\lambda^{2} p^{4}}{48 \beta^{2} k^{4}}, \quad g_{2}=\frac{\lambda^{3} p^{6}}{13824 \beta^{3} k^{6}}
$$

\section{The (2+1)-dimensional modified Novikov-Veselov equations}

Consider the (2+1)-dimensional modified Novikov-Veselov equations system[19,22,23]

$$
\begin{aligned}
& u_{t}+u_{x x x}+u_{y y y}+3 u_{x} w_{x x}+3 u_{y} w_{y y}-u_{x}^{3}-u_{y}^{3}=0, \\
& w_{x y}=u_{x} u_{y} .
\end{aligned}
$$

which passes the three soliton test [22] and the bilinear Painleve test[23]. Recently Chou[17,19] used the Hirota bilinear method to obtain the doulby periodic solutions in terms of theta functions. Here we use the above method to consider their periodic solutions in terms of Weierstrass elliptic function.

Solution 1:

$$
\begin{gathered}
u_{1}=\int^{\xi} \sqrt{-\wp\left(\xi ; g_{2}, g_{3}\right)-\frac{\lambda}{3 k^{2}\left(1+l^{3}\right)}} d \xi \\
w_{1}=-\int^{\xi} \int^{\xi} \wp\left(\xi ; g_{2}, g_{3}\right) d \xi d \xi-\frac{\lambda}{6 k^{2}\left(1+l^{3}\right)} \xi^{2}+C_{1} \xi+C_{2},
\end{gathered}
$$


where $C_{1}, C_{2}, k, l, \lambda$ are constants, $\xi=k(x+l y+\lambda t)$ and $g_{3}$ satisfies

$$
g_{3}=\frac{-4 \lambda^{3}+9 \lambda g_{2} k^{4}\left(1+l^{3}\right)^{2}}{27 k^{6}\left(1+l^{3}\right)^{3}},
$$

Solution 2:

$$
\begin{gathered}
u_{2}=\int^{\xi} \frac{b_{1}}{\sqrt{\frac{3 k^{2}\left(1+l^{3}\right) B}{\lambda} \wp \wp\left(\xi ; g_{2}, g_{3}\right)+B}} d \xi, \\
w_{2}=\int^{\xi} \int^{\xi} \frac{\lambda b_{1}^{2}}{3 k^{2}\left(1+l^{3}\right) B \wp\left(\xi ; g_{2}, g_{3}\right)+\lambda B} d \xi d \xi+C_{1} \xi+C_{2},
\end{gathered}
$$

where $C_{1}, C_{2}, b_{1}, B, k, l, \lambda$ are constants, $\xi=k(x+l y+\lambda t)$ and $g_{2}, g_{3}$ satisfy

$$
g_{2}=\frac{4 b_{1}^{2} \lambda k^{2}\left(1+l^{3}\right)+4 \lambda^{2} B}{3 k^{4}\left(1+l^{3}\right)^{2} B}, \quad g_{3}=\frac{6 b_{1}^{2} \lambda^{2} k^{2}\left(1+l^{3}\right)+8 \lambda^{3} B}{27 k^{6}\left(1+l^{3}\right)^{3} B} .
$$

Solution 3:

$$
\begin{gathered}
u_{3}=\int^{\xi} \frac{-\wp\left(\xi ; g_{2}, g_{3}\right)-\frac{2 \lambda+6 k^{2} a_{1} b_{1}\left(1+l^{3}\right)}{6 k^{2} a_{1} b_{1}\left(1+l^{3}\right)}}{\sqrt{-\wp\left(\xi ; g_{2}, g_{3}\right)-\frac{2 \lambda+12 k^{2} a_{1} b_{1}\left(1+l^{3}\right)}{6 k^{2} a_{1} b_{1}\left(1+l^{3}\right)}}} d \xi, \\
w_{3}=\int^{\xi} \int^{\xi} \frac{\left(-\wp\left(\xi ; g_{2}, g_{3}\right)-\frac{2 \lambda+6 k^{2} a_{1} b_{1}\left(1+l^{3}\right)}{6 k^{2} a_{1} b_{1}\left(1+l^{3}\right)}\right)^{2}}{-\wp\left(\xi ; g_{2}, g_{3}\right)-\frac{2 \lambda+12 k^{2} a_{1} b_{1}\left(1+l^{3}\right)}{6 k^{2} a_{1} b_{1}\left(1+l^{3}\right)}} d \xi d \xi+C_{1} \xi+C_{2},
\end{gathered}
$$

where $C_{1}, C_{2}, a_{1}, b_{1}, k, l, \lambda$ are constants, $\xi=k(x+l y+\lambda t)$ and $g_{2}, g_{3}$ satisfy

$$
\begin{gathered}
g_{2}=\frac{132 a_{1}^{2} b_{1}^{2} k^{4}\left(1+l^{3}\right)^{2}+48 \lambda a_{1} b_{1} k^{2}\left(1+l^{3}\right)+4 \lambda^{2}}{3 k^{4}\left(1+l^{3}\right)^{2}} \\
g_{3}=\frac{1512 a_{1}^{3} b_{1}^{3} k^{6}\left(1+l^{3}\right)^{3}+828 \lambda a_{1}^{2} b_{1}^{2} k^{4}\left(1+l^{3}\right)^{2}+144 \lambda^{2} a_{1} b_{1} k^{2}\left(1+l^{3}\right)+8 \lambda^{3}}{27 k^{6}\left(1+l^{3}\right)^{3}} .
\end{gathered}
$$

\section{8. (2+1)-dimensional generalized system of modified $\mathrm{KdV}$ equation}

Consider the $(2+1)$-dimensional generalized system of modified KdV equation[19,20]

$$
\begin{aligned}
& u_{t}+\sigma\left(u_{x x x}+3 u^{2} u_{x}-3 u_{x} w\right)+u_{x y y}+2 u_{y} v-u_{x} w=0, \\
& w_{x x}-w_{y y}+\left(u^{2}\right)_{y y}=0, \\
& v_{x x}-v_{y y}-\left(u^{2}\right)_{y y}=0 .
\end{aligned}
$$

Chou[19] used the Hirota bilinear mechanism to obtain the plane soliton solution, the dromion solution and solitoff solution of (45). Here by using the above method we arrive at the Weierstrass elliptic function solutions of (45). 
Solution 1:

$$
\begin{gathered}
u_{1}=\sqrt{-\frac{6 k^{2}\left(\sigma+l^{2}\right)\left(1-l^{2}\right)}{3 \sigma+l^{2}+2 l^{3}} \wp\left(\xi ; g_{2}, g_{3}\right)-\frac{2\left[\lambda-c_{1}(3 \sigma+1)+2 l c_{2}\right]\left(1-l^{2}\right)}{3 \sigma+l^{2}+2 l^{3}}}, \\
v_{1}=-\frac{6 l^{2} k^{2}\left(\sigma+l^{2}\right)}{3 \sigma+l^{2}+2 l^{3}} \wp\left(\xi ; g_{2}, g_{3}\right)-\frac{2 l^{2}\left[\lambda-c_{1}(3 \sigma+1)+2 l c_{2}\right]}{3 \sigma+l^{2}+2 l^{3}}+c_{2}, \\
w_{1}=\frac{6 l^{2} k^{2}\left(\sigma+l^{2}\right)}{3 \sigma+l^{2}+2 l^{3}} \wp\left(\xi ; g_{2}, g_{3}\right)+\frac{2 l^{2}\left[\lambda-c_{1}(3 \sigma+1)+2 l c_{2}\right]}{3 \sigma+l^{2}+2 l^{3}}+c_{1},
\end{gathered}
$$

where $\xi=k(x+l y+\lambda t)$, and $c_{1}, c_{2}, k, l, \lambda, g_{2}$ are constants, $g_{3}$ satisfies

$$
g_{3}=\frac{-4\left[\lambda-c_{1}(3 \sigma+1)+2 l c_{2}\right]^{3}+9 k^{4}\left(\sigma+l^{2}\right)^{2} g_{2}\left[\lambda-c_{1}(3 \sigma+1)+2 l c_{2}\right]}{27 k^{6}\left(\sigma+l^{2}\right)^{3}} .
$$

Solution 2:

$$
\begin{gathered}
u_{2}=\frac{b_{1}}{\sqrt{\frac{3 k^{2}\left(\sigma+l^{2}\right) B}{\lambda-c_{1}(3 \sigma+1)+2 l c_{2}} \wp\left(\xi ; g_{2}, g_{3}\right)+B}} \\
v_{2}=\frac{b_{1} l^{2}}{\frac{3 k^{2} B\left(1-l^{2}\right)\left(\sigma+l^{2}\right)}{\lambda-c_{1}(3 \sigma+1)+2 l c_{2}} \wp\left(\xi ; g_{2}, g_{3}\right)+B\left(1-l^{2}\right)}+c_{2}, \\
w_{2}=-\frac{b_{1} l^{2}}{\frac{3 k^{2} B\left(1-l^{2}\right)\left(\sigma+l^{2}\right)}{\lambda-c_{1}(3 \sigma+1)+2 l c_{2}} \wp\left(\xi ; g_{2}, g_{3}\right)-B\left(1-l^{2}\right)}+c_{1},
\end{gathered}
$$

where $\xi=k(x+l y+\lambda t)$, and $c_{1}, c_{2}, b_{1}, B, k, l, \lambda$ are constants, $g_{2}, g_{3}$ satisfy

$$
\begin{aligned}
& g_{2}=\frac{2 b_{1}^{2}\left[\lambda-c_{1}(3 \sigma+1)+2 l c_{2}\right]\left(3 \sigma+l^{2}+2 l^{3}\right)+12 B\left[\lambda-c_{1}(3 \sigma+1)+2 l c_{2}\right]^{2}\left(1-l^{2}\right)}{9 k^{4} B\left(\sigma+l^{2}\right)^{2}\left(1-l^{2}\right)}, \\
& g_{3}=\frac{2 b_{1}^{2}\left[\lambda-c_{1}(3 \sigma+1)+2 l c_{2}\right]^{2}\left(3 \sigma+l^{2}+2 l^{3}\right)+8 B\left[\lambda-c_{1}(3 \sigma+1)+2 l c_{2}\right]^{3}\left(1-l^{2}\right)}{27 k^{6} B\left(\sigma+l^{2}\right)^{3}\left(1-l^{2}\right)},
\end{aligned}
$$

Solution 3:

$$
\begin{gathered}
u_{3}=\frac{\frac{1}{3 \sigma+l^{2}+2 l^{3}}\left[-6 k^{2}\left(\sigma+l^{2}\right)\left(1-l^{2}\right) \wp-N\right]}{\sqrt{\frac{1}{3 \sigma+l^{2}+2 l^{3}}\left[-6 k^{2}\left(\sigma+l^{2}\right)\left(1-l^{2}\right) \wp-M\right]}} \\
v_{3}=\frac{l^{2}\left[-6 k^{2}\left(\sigma+l^{2}\right)\left(1-l^{2}\right) \wp-N\right]^{2}}{\left(1-l^{2}\right)\left(3 \sigma+l^{2}+2 l^{3}\right)\left[-6 k^{2}\left(\sigma+l^{2}\right)\left(1-l^{2}\right) \wp-M\right]}+c_{2}, \\
w_{3}=-\frac{l^{2}\left[-6 k^{2}\left(\sigma+l^{2}\right)\left(1-l^{2}\right) \wp-N\right]^{2}}{\left(1-l^{2}\right)\left(3 \sigma+l^{2}+2 l^{3}\right)\left[-6 k^{2}\left(\sigma+l^{2}\right)\left(1-l^{2}\right) \wp-M\right]}+c_{1},
\end{gathered}
$$

where $\xi=k(x+l y+\lambda t)$, and $c_{1}, c_{2}, a_{1}, b_{1}, k, l, \lambda$ are constants,

$$
\left.N=2\left[\lambda-c_{1}(3 \sigma+1)+2 l c_{2}\right]\left(1-l^{2}\right)+a_{1} b_{1}\left(3 \sigma+l^{2}+2 l^{3}\right)\right],
$$




$$
\left.M=2\left[\lambda-c_{1}(3 \sigma+1)+2 l c_{2}\right]\left(1-l^{2}\right)+2 a_{1} b_{1}\left(3 \sigma+l^{2}+2 l^{3}\right)\right],
$$

and $g_{2}, g_{3}$ satisfy

$$
\begin{gathered}
g_{2}=\frac{1}{27 k^{4}\left(\sigma+l^{2}\right)^{2}\left(1-l^{2}\right)^{2}}\left[72 a_{1} b_{1}\left(1-l^{2}\right)\left[\lambda-c_{1}(3 \sigma+1)+2 l c_{2}\right]\right. \\
\left.+36\left(1-l^{2}\right)^{2}\left[\lambda-c_{1}(3 \sigma+1)+2 l c_{2}\right]^{2}+33 a_{1}^{2} b_{1}^{2}\left(3 \sigma+l^{2}+2 l^{3}\right)^{2}\right], \\
g_{3}=\frac{1}{729 k^{6}\left(\sigma+l^{2}\right)^{3}\left(1-l^{2}\right)^{3}}\left[216\left(1-l^{2}\right)^{3}\left[\lambda-c_{1}(3 \sigma+1)+2 l c_{2}\right]^{3}\right. \\
+648 a_{1} b_{1}\left(1-l^{2}\right)^{2}\left[\lambda-c_{1}(3 \sigma+1)+2 l c_{2}\right]^{2}\left(3 \sigma+l^{2}+2 l^{3}\right) \\
\left.+621 a_{1}^{2} b_{1}^{2}\left(1-l^{2}\right)\left[\lambda-c_{1}(3 \sigma+1)+2 l c_{2}\right]\left(3 \sigma+l^{2}+2 l^{3}\right)^{2}+189 a_{1}^{3} b_{1}^{3}\left(3 \sigma+l^{2}+2 l^{3}\right)\right] .
\end{gathered}
$$

\section{The coupled Klein-Gordon equation}

Consider the coupled Klein-Gordon equation[24]

$$
\begin{aligned}
& \Psi_{x x}-\Psi_{t t}-\Psi+2 \Psi^{3}+2 Q \Psi=0 \\
& Q_{x}-Q_{t}-4 \Psi \Psi_{t}=0 .
\end{aligned}
$$

By using the above method we know that (49) possesses the doubly periodic solutions:

Solution 1:

$$
\begin{gathered}
\Psi_{1}=\sqrt{-k^{2}(1-\lambda)^{2} \wp\left(\xi ; g_{2}, g_{3}\right)-\frac{(2 c-1)(1-\lambda)}{3(1+\lambda)}}, \\
Q_{1}=-2 \lambda k^{2}(1-\lambda) \wp\left(\xi ; g_{2}, g_{3}\right)-\frac{2 \lambda(2 c-1)}{3(1+\lambda)}+c
\end{gathered}
$$

where $\xi=k(x+\lambda t)$, and $g_{3}$ satisfies

$$
g_{3}=\frac{-4(2 c-1)^{3}+9 g_{2} k^{4}\left(1-\lambda^{2}\right)^{2}(2 c-1)}{27 k^{6}\left(1-\lambda^{2}\right)^{3}} .
$$

Solution 2:

$$
\begin{gathered}
\Psi_{2}=\frac{b_{1}}{\sqrt{\frac{3 k^{2}\left(1-\lambda^{2}\right) B}{2 c-1} \wp\left(\xi ; g_{2}, g_{3}\right)+B}} \\
Q_{2}=\frac{2 \lambda b_{1}^{2}}{\frac{3 k^{2}(1-\lambda)\left(1-\lambda^{2}\right) B}{2 c-1} \wp\left(\xi ; g_{2}, g_{3}\right)+B(1-\lambda)}+c,
\end{gathered}
$$

where $\xi=k(x+\lambda t), k, \lambda, b_{1}, c$ are constants, and $g_{2}, g_{3}$ satisfies

$$
g_{2}=\frac{4 b_{1}^{2}(2 c-1)(1+\lambda)+4 B(2 c-1)^{2}(1-\lambda)}{3 B k^{4}\left(1-\lambda^{2}\right)^{2}(1-\lambda)},
$$




$$
g_{3}=\frac{12 b_{1}^{2}(2 c-1)^{2}(1+\lambda)+8 B(2 c-1)^{3}(1-\lambda)}{27 B k^{6}\left(1-\lambda^{2}\right)^{3}(1-\lambda)}
$$

Solution 3:

$$
\begin{gathered}
\Psi_{3}=\frac{-2 k^{2}(1-\lambda)^{2} \wp\left(\xi ; g_{2}, g_{3}\right)-\frac{(2 c-1)(1-\lambda)}{3(1+\lambda)}-a_{1} b_{1}}{\sqrt{-2 k^{2}(1-\lambda)^{2} \wp\left(\xi ; g_{2}, g_{3}\right)-\frac{(2 c-1)(1-\lambda)}{3(1+\lambda)}-2 a_{1} b_{1}}}, \\
Q_{3}=\frac{2 \lambda\left[6 k^{2}\left(1-\lambda^{2}\right)(1-\lambda) \wp\left(\xi ; g_{2}, g_{3}\right)+(2 c-1)(1-\lambda)+3 a_{1} b_{1}(1+\lambda)\right]^{2}}{(\lambda-1)\left[18 k^{2}\left(1-\lambda^{2}\right)^{2} \wp\left(\xi ; g_{2}, g_{3}\right)+3(2 c-1)\left(1-\lambda^{2}\right)+18 a_{1} b_{1}(1+\lambda)^{2}\right]}+c .
\end{gathered}
$$

where $\xi=k(x+\lambda t), k, \lambda, a_{1}, b_{1}, c$ are constants, and $g_{2}, g_{3}$ satisfies

$$
\begin{gathered}
g_{2}=\frac{48 a_{1} b_{1}(2 c-1)(1+\lambda)+4(2 c-1)^{2}(1-\lambda)^{2}+132 a_{1}^{2} b_{1}^{2}(1+\lambda)^{2}}{3 k^{4}\left(1-\lambda^{2}\right)^{2}(1-\lambda)^{2}}, \\
g_{3}=\frac{1}{27 k^{6}\left(1-\lambda^{2}\right)^{3}(1-\lambda)^{3}}\left[8(2 c-1)^{3}(1-\lambda)^{3}+144 a_{1} b_{1}(2 c-1)^{2}(1+\lambda)(1-\lambda)^{2}\right. \\
\left.+828 a_{1}^{2} b_{1}^{2}(2 c-1)(1+\lambda)^{2}(1-\lambda)+1512 a_{1}^{3} b_{1}^{3}(1+\lambda)^{3}\right] .
\end{gathered}
$$

10. The $(2+1)$-dimensional generalization of coupled nonlinear Schrodinger equation

Consider the (2+1)-dimensional generalization of coupled nonlinear Schrodinger equation $[25,26]$

$$
\begin{aligned}
& i \psi_{\tau}+\psi_{\xi \xi}-\psi \chi=0, \\
& i \theta_{\tau}+\theta_{\xi \xi}-\chi \theta=0, \\
& \chi_{\eta}=\left(|\psi|^{2}+\alpha|\theta|^{2}\right)_{\xi} .
\end{aligned}
$$

which was presented by Maccari[25] from Nizhnik equation, where $\psi=\psi(\xi, \eta, \tau)$ and $\theta=$ $\theta(\xi, \eta, \tau)$ are complex and while $\chi=\chi(\xi, \eta, \tau)$ is real. Recently Uthayakumar et al.[26] shew that (53) passed the Painleve test, and gave the bilinear from and one soliton solution. By using the above method we get the doubly periodic solutions of (53)

Solution 1:

$$
\begin{gathered}
\psi_{1}=\exp [i(\alpha \xi+\beta \eta+\gamma \tau)] \sqrt{\frac{2 k^{2} l}{1+\alpha c_{1}^{2}} \wp\left(k(\xi+l \eta-2 \alpha \tau) ; g_{2}, g_{3}\right)-\frac{2 l\left(c_{2}+\alpha^{2}+\gamma\right)}{3\left(1+\alpha c_{1}^{2}\right)}}, \\
\theta_{1}=c_{1} \exp \left[i(\alpha \xi+\beta \eta+\gamma \tau) \sqrt{\frac{2 k^{2} l}{1+\alpha c_{1}^{2}} \wp\left(k(\xi+l \eta-2 \alpha \tau) ; g_{2}, g_{3}\right)-\frac{2 l\left(c_{2}+\alpha^{2}+\gamma\right)}{3\left(1+\alpha c_{1}^{2}\right)}},\right. \\
\chi_{1}=2 k^{2} \wp\left(k(\xi+l \eta-2 \alpha \tau) ; g_{2}, g_{3}\right)-\frac{2\left(c_{2}+\alpha^{2}+\gamma\right)}{3}+c_{2},
\end{gathered}
$$

where $\alpha, \beta, \gamma, k, l, c_{1}, c_{2}$ are constants, and $g_{3}$ satisfies

$$
g_{3}=\frac{-4\left(c_{2}+\alpha^{2}+\gamma\right)^{3}+9 k^{4} g_{2}\left(c_{2}+\alpha^{2}+\gamma\right)}{27 k^{6}} .
$$


Solution 2:

$$
\begin{gathered}
\psi_{2}=\frac{b_{1} \exp [i(\alpha \xi+\beta \eta+\gamma \tau)]}{\sqrt{-\frac{3 k^{2} B}{c_{2}+\alpha^{2}+\gamma} \wp\left(k(\xi+l \eta-2 \alpha \tau) ; g_{2}, g_{3}\right)+B}}, \\
\theta_{2}=\frac{c_{1} b_{1} \exp [i(\alpha \xi+\beta \eta+\gamma \tau)]}{\sqrt{-\frac{3 k^{2} B}{c_{2}+\alpha^{2}+\gamma} \wp\left(k(\xi+l \eta-2 \alpha \tau) ; g_{2}, g_{3}\right)+B}}, \\
\chi_{2}=\frac{b_{1}^{2}\left(1+\alpha c_{1}^{2}\right)\left(c_{2}+\alpha^{2}+\gamma\right)}{-3 k^{2} B l \wp\left(k(\xi+l \eta-2 \alpha \tau) ; g_{2}, g_{3}\right)+B l\left(c_{2}+\alpha^{2}+\gamma\right)}+c_{2},
\end{gathered}
$$

where $\alpha, \beta, \gamma, k, l, c_{1}, c_{2}, b_{1}, B$ are constants, and $g_{2}, g_{3}$ satisfies

$$
\begin{gathered}
g_{2}=\frac{2 b_{1}^{2}\left(c_{2}+\alpha^{2}+\gamma\right)\left(1+\alpha c_{1}\right)+4 B l\left(c_{2}+\alpha^{2}+\gamma\right)^{2}}{3 k^{4} B l}, \\
g_{3}=-\frac{6 b_{1}^{2}\left(c_{2}+\alpha^{2}+\gamma\right)^{2}\left(1+\alpha c_{1}\right)+8 B l\left(c_{2}+\alpha^{2}+\gamma\right)^{3}}{27 k^{6} B l} .
\end{gathered}
$$

Solution 3:

$$
\begin{gathered}
\psi_{3}=\frac{\exp (i Y)\left[\frac{2 k^{2} l}{1+\alpha c_{1}^{2}} \wp\left(X ; g_{2}, g_{3}\right)-\frac{2 l\left(c_{2}+\alpha^{2}+\gamma\right)+3 a_{1} b_{1}\left(1+\alpha c_{1}^{2}\right)}{3\left(1+\alpha c_{1}^{2}\right)}\right]}{\sqrt{\frac{2 k^{2} l}{1+\alpha c_{1}^{2}} \wp\left(X ; g_{2}, g_{3}\right)-\frac{2 l\left(c_{2}+\alpha^{2}+\gamma\right)+6 a_{1} b_{1}\left(1+\alpha c_{1}^{2}\right)}{3\left(1+\alpha c_{1}^{2}\right)}}} \\
\theta_{3}=\frac{c_{1} \exp (i Y)\left[\frac{2 k^{2} l}{1+\alpha c_{1}^{2}} \wp\left(X ; g_{2}, g_{3}\right)-\frac{2 l\left(c_{2}+\alpha^{2}+\gamma\right)+3 a_{1} b_{1}\left(1+\alpha c_{1}^{2}\right)}{3\left(1+\alpha c_{1}^{2}\right)}\right]}{\sqrt{\frac{2 k^{2} l}{1+\alpha c_{1}^{2}} \wp\left(X ; g_{2}, g_{3}\right)-\frac{2 l\left(c_{2}+\alpha^{2}+\gamma\right)+6 a_{1} b_{1}\left(1+\alpha c_{1}^{2}\right)}{3\left(1+\alpha c_{1}^{2}\right)}}} \\
\chi_{3}=\frac{1}{3 l} \frac{6 k^{2} l \wp\left(X ; g_{2}, g_{3}\right)-2 l\left(c_{2}+\alpha^{2}+\gamma\right)-3 a_{1} b_{1}\left(1+\alpha c_{1}^{2}\right)}{6 k^{2} l \wp\left(X ; g_{2}, g_{3}\right)-2 l\left(c_{2}+\alpha^{2}+\gamma\right)-6 a_{1} b_{1}\left(1+\alpha c_{1}^{2}\right)}+c_{2} .
\end{gathered}
$$

where $X=k(\xi+l \eta-2 \alpha \tau), Y=\alpha \xi+\beta \eta+\gamma \tau, \alpha, \beta, \gamma, k, l, c_{1}, c_{2}, a_{1}, b_{1}, B$ are constants, and $g_{2}, g_{3}$ satisfies

$$
\begin{aligned}
g_{2}= & \frac{24 l a_{1} b_{1}\left(c_{2}+\alpha^{2}+\gamma\right)+4 l^{2}\left(c_{2}+\alpha^{2}+\gamma\right)^{2}+33 a_{1}^{2} b_{1}^{2}\left(1+\alpha c_{1}^{2}\right)^{2}}{3 k^{4} l^{2}}, \\
g_{3}= & -\frac{1}{27 k^{6} l^{3}}\left[8 l^{3}\left(c_{2}+\alpha^{2}+\gamma\right)^{3}+72 a_{1} b_{1} l^{2}\left(c_{2}+\alpha^{2}+\gamma\right)^{2}\left(1+\alpha c_{1}^{2}\right)\right. \\
& \left.+207 a_{1}^{2} b_{1}^{2}\left(1+\alpha c_{1}^{2}\right)^{2}\left(c_{2}+\alpha^{2}+\gamma\right)+189 a_{1}^{3} b_{1}^{3}\left(1+\alpha c_{1}^{2}\right)^{3}\right] .
\end{aligned}
$$

It is easy to show that the solution (17) obtain in Ref.[26] is a special case of our solution (54). In addition, when $\xi=\eta$, the system (53) reduces to the coupled nonlinear Schrodinger equation$[25,26]$

$$
\begin{aligned}
& i \psi_{\tau}(\xi, \tau)+\psi_{\xi \xi}(\xi, \tau)-\left(|\psi(\xi, \tau)|^{2}+\alpha|\theta(\xi, \tau)|^{2}\right) \psi(\xi, \tau)=0 \\
& i \theta_{\tau}(\xi, \tau)+\theta_{\xi \xi}(\xi, \tau)-\left(|\psi(\xi, \tau)|^{2}+\alpha|\theta(\xi, \tau)|^{2}\right) \theta(\xi, \tau)=0 .
\end{aligned}
$$


which has many applications in nonlinear optics[27].

According to the relationship between (53) and (57) and the obtained solutions of (53), we can get the corresponding solutions of (57) as

Solution 1:

$$
\begin{aligned}
\psi_{1} & =\exp [i(\alpha \xi+\gamma \tau)] \sqrt{\frac{2 k^{2}}{1+\alpha c_{1}^{2}} \wp\left(k(\xi-2 \alpha \tau) ; g_{2}, g_{3}\right)-\frac{2 l\left(\alpha^{2}+\gamma\right)}{3\left(1+\alpha c_{1}^{2}\right)}}, \\
\theta_{1} & =c_{1} \exp \left[i(\alpha \xi+\gamma \tau) \sqrt{\frac{2 k^{2}}{1+\alpha c_{1}^{2}} \wp\left(k(\xi-2 \alpha \tau) ; g_{2}, g_{3}\right)-\frac{2\left(\alpha^{2}+\gamma\right)}{3\left(1+\alpha c_{1}^{2}\right)}},\right.
\end{aligned}
$$

where $\alpha, \gamma, k, c_{1}$ are constants, and $g_{3}$ satisfies

$$
g_{3}=\frac{-4\left(\alpha^{2}+\gamma\right)^{3}+9 k^{4} g_{2}\left(\alpha^{2}+\gamma\right)}{27 k^{6}} .
$$

Solution 2:

$$
\begin{aligned}
& \psi_{2}=\frac{b_{1} \exp [i(\alpha \xi+\gamma \tau)]}{\sqrt{-\frac{3 k^{2} B}{\alpha^{2}+\gamma} \wp\left(k(\xi-2 \alpha \tau) ; g_{2}, g_{3}\right)+B}}, \\
& \theta_{2}=\frac{c_{1} b_{1} \exp [i(\alpha \xi+\gamma \tau)]}{\sqrt{-\frac{3 k^{2} B}{\alpha^{2}+\gamma} \wp\left(k(\xi-2 \alpha \tau) ; g_{2}, g_{3}\right)+B}},
\end{aligned}
$$

where $\alpha, \gamma, k, c_{1}, b_{1}, B$ are constants, and $g_{2}, g_{3}$ satisfies

$$
\begin{gathered}
g_{2}=\frac{2 b_{1}^{2}\left(\alpha^{2}+\gamma\right)\left(1+\alpha c_{1}\right)+4 B\left(\alpha^{2}+\gamma\right)^{2}}{3 k^{4} B}, \\
g_{3}=-\frac{6 b_{1}^{2}\left(\alpha^{2}+\gamma\right)^{2}\left(1+\alpha c_{1}\right)+8 B\left(\alpha^{2}+\gamma\right)^{3}}{27 k^{6} B} .
\end{gathered}
$$

Solution 3:

$$
\begin{aligned}
\psi_{3} & =\frac{\exp (i Y)\left[\frac{2 k^{2}}{1+\alpha c_{1}^{2}} \wp\left(X ; g_{2}, g_{3}\right)-\frac{2\left(\alpha^{2}+\gamma\right)+3 a_{1} b_{1}\left(1+\alpha c_{1}^{2}\right)}{3\left(1+\alpha c_{1}^{2}\right)}\right]}{\sqrt{\frac{2 k^{2}}{1+\alpha c_{1}^{2}} \wp\left(X ; g_{2}, g_{3}\right)-\frac{2\left(\alpha^{2}+\gamma\right)+6 a_{1} b_{1}\left(1+\alpha c_{1}^{2}\right)}{3\left(1+\alpha c_{1}^{2}\right)}}} \\
\theta_{3} & =\frac{c_{1} \exp (i Y)\left[\frac{2 k^{2}}{1+\alpha c_{1}^{2}} \wp\left(X ; g_{2}, g_{3}\right)-\frac{2 l\left(\alpha^{2}+\gamma\right)+3 a_{1} b_{1}\left(1+\alpha c_{1}^{2}\right)}{3\left(1+\alpha c_{1}^{2}\right)}\right]}{\sqrt{\frac{2 k^{2}}{1+\alpha c_{1}^{2}} \wp\left(X ; g_{2}, g_{3}\right)-\frac{2\left(\alpha^{2}+\gamma\right)+6 a_{1} b_{1}\left(1+\alpha c_{1}^{2}\right)}{3\left(1+\alpha c_{1}^{2}\right)}}},
\end{aligned}
$$

where $X=k(\xi-2 \alpha \tau), Y=\alpha \xi+\gamma \tau, \alpha, \gamma, k, c_{1}, a_{1}, b_{1}, B$ are constants, and $g_{2}, g_{3}$ satisfies

$$
g_{2}=\frac{24 a_{1} b_{1}\left(\alpha^{2}+\gamma\right)+4\left(\alpha^{2}+\gamma\right)^{2}+33 a_{1}^{2} b_{1}^{2}\left(1+\alpha c_{1}^{2}\right)^{2}}{3 k^{4} l^{2}},
$$




$$
\begin{gathered}
g_{3}=-\frac{1}{27 k^{6}}\left[8\left(\alpha^{2}+\gamma\right)^{3}+72 a_{1} b_{1}\left(\alpha^{2}+\gamma\right)^{2}\left(1+\alpha c_{1}^{2}\right)\right. \\
\left.+207 a_{1}^{2} b_{1}^{2}\left(1+\alpha c_{1}^{2}\right)^{2}\left(\alpha^{2}+\gamma\right)+189 a_{1}^{3} b_{1}^{3}\left(1+\alpha c_{1}^{2}\right)^{3}\right] .
\end{gathered}
$$

\section{Summary and Questions}

In summary, based on the Weierstrass elliptic function, we have developed a simple and powerful method, which is called the Weierstrass elliptic function expansion method. With the aid of symbolic computation ( Maple), the method is applied to seek doubly periodic solutions of many nonlinear mathematical physics equations such as the new integrable Davey-Stewartson-type equation, the $(2+1)$-dimensional modified KdV equation, the the generalized Hirota equation in $2+1$ dimensions, the Generalized KdV equation, the $(2+1)$ dimensional modified Novikov-Veselov equations, (2+1)-dimensional generalized system of modified KdV equation, the coupled Klein-Gordon equation, and the $(2+1)$-dimensional generalization of coupled nonlinear Schrodinger equation. As a result, some new doubly periodic solutions are obtained in terms of the Weierstrass elliptic function. Moreover solitary wave solutions and singular solitary wave solutions are also given as simple limits of doubly periodic solutions. The method can be also extend to other types of nonlinear wave equations.

We know that the above-mentioned method is only used to solve these nonlinear ODEs with constant coefficients or nonlinear partial differential equations that can be reduced to be the corresponding ODEs with constant coefficients by using some transformations, otherwise the method will not work. In order to overcome the disadvantage, we change the method into a general form. For given nonlinear differential equation (1), we do not need make the travelling wave transformation. We directly assume that it has the solution in the form

$$
\begin{gathered}
u(x, t)=a_{0}(x, t)+\sum_{i=1}^{n}\left(a_{i}(x, t)\left[A(x, t) \wp\left(\psi(x, t) ; g_{2}, g_{3}\right)+B(x, t)\right]^{\frac{i}{2}}\right. \\
\left.+b_{i}(x, t)\left[A(x, t) \wp\left(\psi(x, t) ; g_{2}, g_{3}\right)+B(x, t)\right]^{-\frac{i}{2}}\right) \\
=a_{0}(x, t)+\sum_{i=1}^{n}\left[a_{i}(x, t)\left(\sqrt{A(x, t) \wp\left(\psi(x, t) ; g_{2}, g_{3}\right)+B(x, t)}\right)^{i}\right. \\
\left.+\frac{b_{i}(x, t)}{\left(\sqrt{A(x, t) \wp\left(\psi(x, t) ; g_{2}, g_{3}\right)+B(x, t)}\right)}\right],
\end{gathered}
$$

where $a_{0}(x, t), a_{i}(x, t), b_{i}(x, t), A(x, t), B(x, t), \psi(x, t)$ are unknown functions of $x, t$, and $\wp\left(\psi(x, t) ; g_{2}, g_{3}\right)$ satisfies

$$
[\wp(\psi(x, t))]^{\prime 2}=4 \wp^{3}(\psi(x, t))-g_{2} \wp(\psi(x, t))-g_{3},
$$

or

$$
[\wp(\psi(x, t))]^{\prime \prime}=6 \wp^{2}(\psi(x, t))-\frac{1}{2} g_{2},
$$

where $g_{2}, g_{3}$ are real parameters and called invariants[1,2]. 
It is easy to see that if all the functions, $a_{0}(x, t), a_{i}(x, t), b_{i}(x, t), A(x, t), B(x, t), \psi(x, t)$, are only constants, then the transformation (61) reduces to (3). Otherwise we may find non-travelling wave types of doubly periodic solutions. They contain more physical information which is used to explain some physical phenomena. This will be considered in future.

\section{References}

[1] D. F. Lawden, Elliptic Functions and Applications, Springer-Verlag, New York, 1989.

[2] E.T. Whittaker and G.N. Watson, A Course of Modern Analysis. Cambridge University Press, London, 1950.

[3] A. V. Porubov, Phys. Lett. A, 221(1996)391.

[4] A. V. Porubov and M. G. Velarde, J. Math. Phys., 40(1999)884.

[5] S. K. Liu, et al., Phys., Lett. A, 289(2001) 69.

[6] Z. Fu, et al.,Phys., Lett. A, 290(2001) 72.

[7] Z. Y. Yan, Commun. Theor. Phys., 38(2002) 143.

[8] Z. Y. Yan, Commun. Theor. Phys., 38(2002) 400; 39(2003) 144.

[9] Z. Y. Yan, Chaos, Solitons, and Fractals, 15(2003) 575.

[10] Z. Y. Yan, Comput. Phys. Commun., 148(2002) 30.

[11] Z. Y. Yan, Chaos, Solitons and Fractals,( to appear)

[12] A. Maccari, J. Math. Phys., 40(1999) 3971.

[13] B. Konopelchenko and V. Dubrovsky, Phys. Lett. A, 102(1984) 15.

[14] K. Toda, J. Nonlinear Math. Phys. (Supplement), 9(2002) 207.

[15] A. Maccari, J. Math. Phys., 39(1998) 6547.

[16] Z. Y. Yan, J. Phys. A, 36(2003) 1961.

[17] K. W. Chou, Wave Motion, 35(2002) 71.

[18] C. T. Yan, Wave Motion, 26(1997) 219.

[19] K.W. Chou, J. Phys. Soc. Jpn., 65(1996) 1971; 72(2003) 273.

[20] M. Boiti., et al., Phys. Lett. A, 132(1988) 432.

[21] Z. Y. Yan, Phys. Lett. A, ( to submit).

[22] J. Hietarinta, J. Math. Phys., 28(1987) 2094

[23] J. J. C. Nimmo, Applications of Analytic and Geometric Methods to Nonlinear Differential Equations, ed. P.A. Clarkson, Kluwer Academic Press, 1993, p.183.

[24] K. Porsezian and T. Alagesan, Phys. Lett. A, 198(1995) 378.

[25] A. Maccari, J. Math. Phys., 38(1997) 4151.

[26] A. Uthayakumar, et al., Chaos, Solitons and Fractals, 10(1999) 1513

[27] S.V. Manakov, Sov. Phys. JETP, 38(1974) 248. 